$10.2478 / \mathrm{gb}-2021-0007$

sciendo

\title{
Zwischen Fiktion und historischer Realität: Gusel Jachinas Roman Wolgakinder
}

\footnotetext{
Sunhild GALTER

Doz. Dr., Lucian-Blaga-Universität Sibiu/Hermannstadt; E-Mail: sunhild.galter@ulbsibiu.ro
}

\begin{abstract}
Gusel Jachina is a Russian writer. Her grandfather, a former German teacher in one of the villages along the Volga River, founded by German colonists, inspired her second novel "Wolgakinder" (Children of the Volga). She presents over 20 years of eventful history as it is seen by Jakob Bach, a German teacher in the village Gnadental on the banks of the Volga. It is an opulent novel of 600 pages, written in a rather baroque style, trying to not only present historic events from the beginning of the Soviet era but to recreate the atmosphere of those years full of Ups and Downs not only for the German speaking population.
\end{abstract}

Key words: Gusel Jachina, "Wolgakinder", Gnadental on the Volga River, events from the beginning of the Soviet era.

Der Roman Wolgakinder, von dem im Folgenden die Rede sein soll, hat auf den ersten Blick wenig mit dem Bereich der Germanistik gemeinsam. Denn die Autorin Gusel Jachina ist eine auf Russisch schreibende Tatarin. Geboren wurde sie am 1. Juni 1977 in Kasan, der Hauptstadt der autonomen Republik Tatarstan in Russland. Diese mit 1,24 Millionen Einwohnern sechstgrößte Stadt Russlands liegt an der Wolga.

Und damit gibt es einen ersten Berührungspunkt, denn an der Wolga entstanden zur der Zeit und auf Wunsch der Zarin Katharina die Große zwischen 1764 und 1767 hundertvier von bayerischen, badischen, rheinischen, pfälzischen und hessischen 
Kolonisten gegründete deutsche Siedlungen. Die deutsche Bevölkerung an der unteren Wolga bekam bald den Namen „die Wolgadeutschen“. Sie sollten die Steppe urbar machen und die Einfälle der Tataren abwehren. Bis 1874 hatten sie Sonderprivilegien, wie z.B. Benutzung der deutschen Verwaltungssprache, administrative Autonomie u. Ä. Dadurch konnten die Wolgadeutschen über zwei Jahrhunderte hinweg ihre Muttersprache, ihre Bräuche, ihre Märchen, Sagen und Sprichwörter bewahren, wobei letztere in dem Roman zeitweilig eine tragende Rolle spielen. „Der gute Boden brachte Rekordernten. Selbst Lenin war beeindruckt von den Früchten ihres Fleißes und ihren schmucken bunten Häusern. Er gewährte ihnen einen Sonderstatus als quasi selbständige Republik, ein Vorbild für alle anderen sollten sie sein. Doch mit der Alleinherrschaft Stalins brachen alle Vorrechte weg. "1

Nachdem Stalin 1921 die gesamte Ernte beschlagnahmt und für seine Zwecke benutzt hatte, kam es 1921/22 zu einer großen Hungersnot, bei der Hunderttausende starben. Als eine Art Wiedergutmachung gab es daraufhin für eine kurze Zeit, und zwar zwischen 1924 und 1941, sogar eine Sozialistische Sowjetrepublik der Wolgadeutschen. 1941 jedoch, nach dem Angriff Deutschlands auf die Sowjetunion wurden dann auf Stalins Befehl etwa 400.000 Wolgadeutsche als so genannte Kollaborateure nach Sibirien und Zentralasien zwangsumgesiedelt.

Die Autorin veranschaulicht in ihrem umfangreichen Werk einen guten Teil der ersten Hälfte des 20. Jahrhunderts, was mit Hilfe zahlreich eingesetzter Zeitraffungen und Zeitsprünge auch ziemlich gut gelingt, obwohl die Leser manchmal schon

${ }^{1}$ Frisé, Maria: Unterrichte meine Tochter, aber sieh sie nicht an! Aufwachsen in entsetzlichen Zeiten: Gusel Jachinas Roman „Wolgakinder“. In: Frankfurter Allgemeine Zeitung vom 14.08.2019, unter https:// www.buecher.de/shop/sowjetunion/wolgakinder/jachina-gusel/products_products/detail/prod_id/56247477/\#reviews (abgerufen am 31.10.2020) 
zurückblättern müssen, um sich die Zusammenhänge zu vergegenwärtigen. ,Gusel Jachina [kehrt] zurück in die Anfänge der Sowjetunion und die Zeit davor. Der allgegenwärtige zeitgeschichtliche Hintergrund sind die Jahre 1916 bis 1938, als der Erste Weltkrieg, die Oktoberrevolution, der nachfolgende Bürgerkrieg, Hungersnöte, Zwangskollektivierung und der sogenannte Große Terror Millionen Menschen das Leben kostet."2

Diese zeitgeschichtlichen Ereignisse bilden das Thema des bisher zweiten Romans Gusel Jachinas, in deutscher Übersetzung erschienen im Sommer 2019 unter dem Titel Wolgakinder. Schon in ihrem Debütroman Suleika öffnet die Augen ${ }^{3}$ geht es um die Deportationen der missliebigen Minderheiten vorzugsweise in die Arbeitslager in Sibirien. In Jachinas erstem Roman Fall geht es um ihre tatarische Großmutter, die deportiert worden war. Auch war einer ihrer Großväter Deutschlehrer in den Ortschaften an der Wolga und vermittelte Jachina die Liebe zur deutschen Sprache und Kultur, die sie dann in ihrem Studium wissenschaftlich vertieft hat. Denn Gusel Jachina ist studierte Germanistin $^{4}$ und Anglistin, aber auch Filmemacherin ${ }^{5}$.

Dem faszinierenden, mythisch aufgeladenen Flusslauf der mächtigen Wolga, die das Leben der Menschen an ihren Ufern seit Urzeiten begleitet und Zeuge gewaltiger historischer Umbrüche wurde, nähert sich die Autorin deshalb auch eher mit dem Blick und der Perspektive des Films. Hilfreich bei dieser Herangehensweise ist ,die Ausbildung an der Moskauer

${ }^{2}$ Hochweis, Olga: Das traurige Leben des Jakob Iwanowitsch Bach. Deutschlandfunk Kultur vom 1.10.2019, unter: https://www.deutschlandfunkkultur.de/gusel-jachina-wolgakinder-das-traurige-lebendes-jakob.950.de.html?dram:article_id=460032 (abgerufen am 31.10.2020)

3 Jachina, Gusel: Suleika öffnet die Augen. Berlin 2017.

${ }^{4}$ Gusel Jachina studierte an der Kasaner Tatarischen Staatlichen Geisteswissenschaftlich-Pädagogischen Universität Germanistik und Anglistik.

${ }^{5}$ Im Jahre 2015 beendete sie auch ihre Ausbildung an der staatlichen Moskauer Filmhochschule, am Gerassimow-Institut für Kinematografie mit dem Diplom. 
Filmschule: Jachina erzählt über weite Strecken im filmischen Präsens, versteht sich auf dramatische Wendungen und lässt den Point of View elegant von den Protagonisten ,auf weitere Figuren hinübergleiten." ${ }^{\text {"6 }}$

Die Sprache ist außerordentlich bildreich, zuweilen fast schon zu bildreich. Dabei werden alle Sinne des Betrachters bzw. des Lesers angesprochen, die geschilderten Orte erwachen vor dem inneren Auge zum Leben, man meint die unterschiedlichsten Gerüche zu riechen, die vielfältigsten Geräusche zu hören. Das alles trägt dazu bei, dass auch die geschilderten Gewaltszenen, der Hunger, der Verfall, die räuberischen Überfälle sehr eindringlich wirken und manche lang zurückliegenden, jedoch historisch verbrieften Ereignisse unmittelbar miterlebt werden können. ${ }^{7}$ Dabei übertreibt die Autorin zuweilen und manche der barock-opulenten Formulierungen können als nachgerade kitschig bezeichnet werden, was aber anteilmäßig bei dem sich über fast 600 Seiten erstreckenden Epos vernachlässigbar ist. Auch werden sie durch die feine Ironie, die herauszulesen ist, wieder relativiert und abgemildert.

Schulmeister Bach war einer unheilvollen Leidenschaft verfallen [...]: er liebte Gewitter. Nicht wie ein friedlicher Maler oder ein ehrbarer Dichter vom Fenster her dem Tosen der Naturgewalten zuschaut, sich an dem rollenden Donner und den grellen Farben eines Unwetters ergötzt. O nein! Bach war Gewittern verfallen wie ein armer Säufer dem Wodka aus Kartoffelschalen oder ein Morphiumsüchtiger seiner Droge. (...)

Am Himmel, der von dicken Wolken so angeschwollen war, dass er fast bis zur Erde reichte, erhob sich ein Rauschen, ein Krachen und

${ }^{6}$ Großschupf, Johannes: Die Liebe in Sibirien. Der Tatarin Gusel Jachina ist mit „Suleika öffnet die Augen“ ein kraftvolles Debüt gelungen. In: Literaturkritik.de Rezensionsforum vom 4. April 2017, unter: https:// literaturkritik.de/jachina-suleika-oeffnet-augen-liebe-sibirien-tataringusel-jachina-ist-mit-suleika-oeffnet-augen-ein-kraftvolles-debuetgelungen,23153.html (abgerufen am 1.11.2020)

7 Vgl. Olga Hochweis, 2019. 
ohrenbetäubendes Dröhnen. Dann wurde es plötzlich taghell, und mit stumpfem Stöhnen stürzte eine wahre Wasserflut herab - ein Gewitterguss begann. Bach riss den Hemdkragen auf, entblößte die schmächtige Brust, warf den Kopf zurück und öffnete den Mund. Ströme von Wasser klatschten auf seinen Körper, umspülten ihn ganz und gar, die Füße spürten, wie die Erde bei jedem Donnerschlag erbebte. Blitze - gelbe, blaue und schwarz-violette zuckten immer schneller herab - über seinem Kopf oder tief in seinem Inneren. Das Brodeln in seinen Muskeln steigerte sich zu solcher Heftigkeit, das ihm war, als müsste der nächste Schlag vom Himmel seinen Körper in tausend winzige Stücke zerfetzen und in der Steppe verstreuen. ${ }^{8}$

Die äußere Handlung des Romans kann relativ schnell erzählt werden. Sie setzt im Jahr 1916 ein und spielt sich weitestgehend in dem Dorf Gnadental, einer von Steppengebieten umgebenen deutschen Siedlung am Unterlauf der Wolga, ab. Hier wirkt der zweiunddreißigjährige Dorflehrer Jakob Iwanowitsch Bach, ein magerer, ungepflegter Sonderling mit autistischen Zügen, der stundenlang Goethe und Schiller rezitieren könnte, aber kaum überlebensfähig ist. Er kennt zwar alle Dorfbewohner ganz genau, da er sie scharf beobachtet und ihr Wesen analysiert, aber er kann nicht mit ihnen und nicht mit den etwa 70 Kindern, die er unterrichten soll, kommunizieren oder sozial interagieren. Die Schule verfügt über einen großen Klassenraum und einen kleinen Wohnraum für den Lehrer. „Es ist eine vormoderne Welt, die Jachina mit tausend Details und ethnologischem Eifer ausstattet. Der durchschnittliche Gnadentaler ist in Bachs Worten ,ein unverbesserlicher Fatalist, fromm und abergläubisch', dazu ,allem Neuen, jedem Fortschritt und jedem Experiment abhold'. Wo sonst ließe sich der Aufprall der bolschewistischen Umwälzungen eindrucksvoller demonstrieren als an diesem ,Ort am Rande der Gegenwart"?" Jachina baut

8 Jachina 2019, S. 22-23.

9 Zekri, Sonja: Im Kopf von Josef Stalin. Gusel Jachinas Roman „Wolgakinder“. In: Süddeutsche Zeitung vom 17.01.2020, unter: https://www.buecher.de/shop/sowjetunion/wolgakinder/jachina-gusel/ 
die historischen Daten über das Leben und die Traditionen der Wolgadeutschen sehr detailliert in ihren Roman ein, der auch mit einem umfangreichen Anhang an Anmerkungen mit geschichtlichem Hintergrundmaterial versehen ist.

Die mythische Topografie, die Jachina zum Schauplatz der Handlung macht - zwei Welten, durch einen mächtigen Strom voneinander getrennt -, und die archetypischen Figuren lassen den Roman parabelhaft wirken, obwohl er auf eine reale Zeitund Familiengeschichte gründet. Bach, der in dem von einer weiten Steppenlandschaft umgebenen Gnadental an dem flachen Ufer der Wolga lebt, wird kurz vor der Russischen Revolution bzw. den Revolutionen des Jahres 1917 von einem reichen Gutsbesitzer an das andere Wolga-Ufer einbestellt, um dessen siebzehnjährige Tochter Klara zu unterrichten und damit für eine Ehe ,im Reich“ heiratsfähig zu machen. Erst lehnt er ab, denn noch nie war jemand aus dem Dorf auf der anderen Seite des Flusses gewesen, schon die Überquerung ist gefährlich, die Steilufer scheinen unüberwindliche Hindernisse zu sein. Doch der kirgisische Bote setzt sich durch, Bach folgt ihm und erreicht zu seiner Verwunderung einen mitten in ausgedehnten Wäldern liegenden reichen Gutshof, auf dem lediglich der Besitzer Udo Grimm, seine Tochter Klara und deren Kinderfrau Tilda mit einigen namenlosen kirgisischen Knechten leben. Durch Tilda und Klara lernt Bach die schmucklosen, im Dialekt erzählten Märchen und Sagen kennen, die später ihm und der kleinen Anna das Leben retten werden. Die zwei verlieben sich ineinander, obwohl Klara beim Unterricht hinter einem Wandschirm sitzen muss. Bach hat jemanden gefunden, der noch hilfloser und verletzlicher ist als er selbst und Klara ist von seiner Stimme und der gewählten Sprache beeindruckt.

Als ihr Vater nach einem Jahr Unterricht meint, er könne ihr nun in Deutschland einen Ehemann finden, reist er mit Klara

products_products/detail/prod_id/56247477/\#reviews (abgerufen am 1.11.2020) 
und Tilda ab, die Kirgisen werden entlassen, der Hof dichtgemacht. Doch Klara läuft bei dem ersten Bahnhofsaufenthalt davon und kehrt zu Bach nach Gnadental zurück. Weil Klara keine Papiere hat und sie auch niemand weit und breit kennt, können sie nicht heiraten. Um dem immer aggressiveren Gerede und Verhalten der Gnadentaler zu entgehen, überqueren sie den Fluss und lassen sich auf dem Gutshof nieder, den sie notdürftig wieder in Betrieb nehmen. Doch Bach lernt im Laufe der Zeit alle physisch anstrengenden Arbeiten auszuführen und während außerhalb ihres magischen Waldes und jenseits der großen Wolga Geschichte geschrieben wird, Bürgerkriege, Enteignungen, Auswanderungswellen das ehemals reiche Gnadental zu einer Dorfruine verkommen lassen, leben die zwei in der Idylle des Bauernhofes zufrieden miteinander.

Im Herbst, als die Steppe von der Sonne verbrannt und von Explosionen zerwühlt war, die Wälder am rechten Wolgaufer hingegen in allen Tönen von Gelb und Rot erglühten, tauchten Geschwader von Schiffen und Booten auf. (...) Manche waren beschädigt, hatten einen aufgerissenen Bauch oder ein zerschmettertes Rückgrat. (...) Bach und Klara beobachteten die Vorgänge von der Steilküste aus. Sie verstanden überhaupt nichts. Wahrscheinlich war das der Krieg. ${ }^{10}$

Der Schauplatz weiter Teile des Romans mutet märchenhaft und mythisch an. Er erinnert an das Waldhaus vieler deutscher Märchen. „Ein Ort, der zeitlos erscheint. Und ein Ort, an dem man die grausame Vergangenheit nur durch eine Art Fernglas beobachtet."11

Zum vollkommenen Glück fehlt nur ein Kind, aber sie bleiben jahrelang kinderlos. Nach der Vergewaltigung durch drei

${ }^{10}$ Jachina 2019, S. 113.

${ }^{11}$ Grützner, Annika: Ein Leben in der gewählten Einsamkeit. „Wolgakinder" von Gusel Jachina. In: Read Ost. Literatur Blog vom 23. Juni 2019, unter: https://read-ost.com/2020/06/23/ein-leben-in-der-gewahlten-einsamkeit-wolgakinder-von-gusel-jachina/ (abgerufen am 1.11.2020) 
Kirgisen, die auf der Suche nach Essbarem auf den Hof stoßen, ist Klara endlich schwanger, während Bach durch das traumatische Erlebnis seine Stimme verliert, sein wichtigstes Charakteristikum. Deshalb führt er nun Buch über die Ereignisse. Bei den seltenen nächtlichen Besuchen in Gnadental, am anderen Wolgaufer, erlebt er den Verfall des Dorfes mit und benennt die Jahre nach den auffälligsten Ereignissen: „Das Jahr der verwüsteten Häuser“, die Jahre ohne richtigen Sommer nennt er „Jahre des ewigen Novembers", das Jahr, als der Krieg Gnadental erreicht „Jahr des Wahnsinns“, das Jahr der Beschlagnahmung des Viehs und das Vertreiben der Großbauern „Jahr der ungeborenen Kälber“, es folgt das „Jahr der Hungernden“" usw.

Klara verblutet bei der Geburt und die zarte Frau, die schon zu Lebzeiten eine Schneewittchen-Existenz in den undurchdringlich scheinenden Wäldern des rechten Wolgaufers geführt hat, zuerst allein mit Vater und Kinderfrau, dann mit Bach, wird im Eishaus, ihrem ,gläsernen“ Sarg, allmählich zur mythischen Erinnerung.

Bach zieht das kleine Mädchen Anna alleine und sprachlos groß. Erst stiehlt er bei Nacht Ziegenmilch aus dem Dorf, doch dann wird er dabei ertappt. Die schicksalhafte Fügung will es, dass der für die Verwaltung eingesetzte kommunistische Kommissar ein besonderes Interesse am Volksgut der Wolgadeutschen hat. Bachs durch Klara erworbenes Wissen rettet ihn.

Denn der Schulmeister soll alles, was er kennt an Sprichwörtern und Brauchtum, später auch an Märchen, aufschreiben. Der Genosse ist ein Sammler. Er bezahlt in Naturalien, bitter nötig, um den Dorfschulmeister und die kleine Anna am Leben zu erhalten. Ein zweites Kind, ein verwahrloster (kirgisischer) Junge, läuft ihm zu. Nun sind sie zu dritt, und Bach fühlt sich berufen, die beiden jungen Menschen auf ein Leben in der unruhigen Welt vorzubereiten. ${ }^{12}$

${ }^{12}$ Frisé 2019. 
Die kommunistische Verwaltung des Bezirks sammelt alle nicht eingeschulten Kinder ein und entdeckt auch Anna und Wossja. Letztendlich kommen die Kinder in ein Schulinternat der relativ nahe gelegenen Kleinstadt Saratow, wo Bach sie regelmäßig besucht, bis er verhaftet und in ein Arbeitslager nach Kasachstan gebracht wird, wo er bei einem Grubenunglück umkommt.

Anna wird 1941 mit den anderen Wolgadeutschen nach Kasachstan deportiert, verliert bei einem Unfall ein Bein und lebt anschließend dort als Buchhalterin, bis sie ihr Kindheitsgefährte Wossja nach dem Krieg, an dem er als Freiwilliger teilgenommen hat, aufspürt. Sieheiraten und Wassili Wassiljewitsch Wolgin wird an einer Dorfschule Deutschlehrer, so wie es sein Ziehvater in Gnadental gewesen war. Damit schließt sich der Kreis.

Es sind immer wieder märchenhafte Fügungen und Zufälle, die Spannung aufbauen und die Handlung vorantreiben. Gusel Jachina mischt sie sorglos mit legendenhaften Elementen und historisch Verbrieftem. Ein sehr farbiges Sprachgewebe entsteht auf diese Weise, das sich mitunter zum tragischen Epos der Wolgadeutschen verdichtet. ${ }^{13}$

Einen besonderen Stellenwert im Roman nimmt die Wolga ein, als Lebensader der Region, als letzte Ruhestätte zahlreicher Verunglückten, Ermordeten, Verhungerten.

Die Wolga teilte die Welt in zwei Hälften.

Das linke Ufer war niedrig und gelb; flach lag es da und ging in die Steppe über, aus der jeden Morgen die Sonne heraufstieg. Die Erde schmeckte bitter und war von Zieselmäusen durchwühlt, das Gras wuchs dicht und hoch, die Bäume hingegen waren niedrig und selten. Getreide- und Melonenfelder bis zum Horizont, farbenprächtig wie eine baschkirische Bettdecke. Am Ufer klebten Dörfer. Der Steppenwind war heiß und würzig, er roch nach den Wüsten Turkmeniens und dem Salz des Kaspischen Meers.

${ }^{13}$ Ebenda. 
Wie es am anderen Ufer aussah, wusste niemand. Über der rechten Seite des Flusses erhoben sich mächtige Berge, die senkrecht, wie mit einem Messer geschnitten, in den Fluss stürzten. An der Kante rieselte zwischen den Steinen Sand herunter, doch die Berge sanken nicht ein, sie wurden Jahr um Jahr nur steiler und fester - von blaugrünem Wald bedeckt im Sommer und tiefverschneit im Winter. Hinter diesen Bergen ging die Sonne unter. ${ }^{14}$

In einer wohl als Schlüsselszene gedachten Episode treibt Bach in der Wolga, dem mächtigen Fluss, der in seinen mächtigen Tiefen Zeugnis ablegt von menschlichen Schicksalen, historischen Ereignissen, Krieg, Kollektivierung, Vertreibung und Deportation und damit jedes Einzelschicksal relativiert. Bach hat nach Klaras Vergewaltigung seine Stimme verloren, was durchaus keine Metapher ist, sondern als Folge traumatischer Erlebnisse medizinisch belegt ist. Er weicht auf die Schriftsprache aus und wird zum Geschichtenerzähler, um Annas Überleben zu sichern. Erst schreibt er dem kommunistischen Funktionär, der nun dem Dorf vorsteht, die von Klara gehörten Märchen und Sagen auf, und als die ihm ausgehen, erfindet er immer neue, übertriebenere Geschichten. Nach seiner Vision in den Wassern der Wolga sieht er ein, dass der Einzelne nur ein kleines Rädchen im Ablauf der Geschichte ist, und gibt jeden persönlichen Einsatz auf. Durch seine berichterstattenden Hefte wird er zum unbeteiligten Chronisten der Ereignisse in Gnadental.

Die Kritik betrachtet den Roman nicht immer wohlwollend. Die zuweilen überschwängliche Sprache, die barocken Bilder, wie sie zum Beispiel Bach auf seiner Höllenfahrt durch die Wolga begleiten, die naturmagische symbolische Aufladung des Flusses, das alles sind Elemente, die bei den deutschen Rezensenten zum Teil auf Unverständnis stoßen. In der nicht gezeichneten Besprechung in den Westfälischen Nachrichten wird die Eindimensionalität des Schauplatzes und der Figuren

${ }^{14}$ Jachina 2019, S. 9. 
und die oft als floskelhaft empfundene Sprache bemängelt, das Fazit lautet, dass ,eine reizvolle Anfangsidee, die Kombination aus Welt- und Dorfhistorie, im Roman etwas auf der Strecke geblieben [ist]. '15

Dem kann man einiges entgegenhalten, so zum Beispiel, dass der Schulmeister Bach in seinem kleinen Dorf an der Wolga und später dann in der Einöde der fast endlosen Wälder wohl kaum Gelegenheit hat, eine größere Entwicklung durchzumachen. MeinesErachtens istdurchaus eineEntwicklungslinie zu erkennen. Bach, der bis zur Begegnung mit Clara nur auf sich selbst fixiert ist, der den privaten Umgang mit den Dorfbewohnern und einen persönlichen Bezug $\mathrm{zu}$ seinen Schülern scheut, öffnet sich langsam und zögerlich Clara gegenüber, und als er sie verliert, überträgt er alle tief im Inneren begrabenen Gefühle auf das Neugeborene, auf Anna, deren Leben und Überleben er hinfort zu seinem Lebensziel macht. Dermaßen vorbereitet, kann er, dem das Schicksal seiner Schüler vollkommen gleichgültig gewesen war, auch für den namenlosen, ums Überleben kämpfenden Jungen, der wie viele andere auf der Suche nach etwas Essbarem und auf der Flucht vor den Behörden entlang der Wolga streunt, fürsorgliche Gefühle entwickeln und sogar so etwas wie eine Vaterfigur für ihn werden.

Tatsächlich mutet jedoch der Einschub, in dem es um Josef Stalins Besuch in der Wolgaregion geht, als zusammenhanglos eingefügte Episode an. Sonja Zekri meint dazu in ihrer Rezension in der Süddeutschen Zeitung:

Dass sich Jachina, die sonst auf jede Psychologisierung verzichtet, ausgerechnet in den Kopf Josef Stalins denkt, begreift man dann gar nicht mehr. Seitenlang geht Stalin beim Billard seine strategischen Chancen gegen Hitler durch und kommt am Ende zu erfreulichen Ergebnissen. Dies aber ist eine Metaphorik von solcher Schlichtheit,

${ }^{15}$ o. A.: Keine Gnade in Gnadental: Gusel Jachinas „Wolgakinder“. 2019. 
dass man die Szene selbst dann nicht glauben will, wenn sie sich wirklich so zugetragen hätte. ${ }^{16}$

Trotz aller erkennbaren Schwächen beim Versuch, die historische Realität, und zwar gleich den jahrzehntelang dauernden Aufbau, Aufstieg und Verfall einer ganzen Region zu fiktionalisieren, sind weite Teile sehr gut gelungen. Auch der grandios ausholende, vor rhetorischen Mitteln strotzende Schreibstil scheint heutigen Lesern unangemessen, veraltet, und dennoch wäre diesem Thema anders nicht beizukommen. Zu gewaltig ist die von der Natur gegebene Szenerie, zu gewaltig sind die geschilderten historischen Umwälzungen. Jenseits aller Unterschiede in der Bewertung dieses Romans durch die Kritik bleibt er ein wertvoller Einblick in die Gedankenwelt der einzelnen, die von der Geschichte überrollt werden.

Zum Abschluss Jachinas eigene Worte, was nicht nur sie zum Schreiben darüber brachte, sondern auch viele beim Lesen faszinierend empfinden:

Es war eine absolut ungewöhnlich Zeit. Auf der einen Seite war es eine Zeit voller Tragödien, Millionen Menschen starben. Erst die Revolution, dann der Bürgerkrieg, dann Hunger Anfang der 20er Jahre, dann der Hunger Anfang der 30er, dann der "Große Terror“ 1937/38, dann der „Große Vaterländische Krieg“ und die Deportationen ganzer Völker. Auf der anderen Seite war diese Zeit auch voller Euphorie, besonders die 20er Jahre. Viele Menschen glaubten tatsächlich innigst, dass sie eine großartige Zukunft aufbauen werden. Und diese Mischung aus Tragödie und gleichzeitiger Blüte, etwa im kulturellen Bereich, sie ist sehr anziehend, sie fasziniert mich. ${ }^{17}$

${ }^{16}$ Zekri 2020.

${ }^{17}$ Funk, Victor/ Gusel Jachina: Autorin Gusel Jachina: „Das ist das Echo der Vergangenheit, das sich in uns zeigt". Interview in Frankfurter Rundschau, 6.05.2020, auch unter: https://www.fr.de/kultur/literatur/ gusel-jachina-russland-stalin-echo-vergangenheit-zr-13750778.html (abgerufen am 1.11.2020) 


\section{Literaturverzeichnis:}

\section{Primärliteratur:}

Jachina, Gusel: Suleika öffnet die Augen. Berlin: Aufbau 2017. Jachina, Gusel: Wolgakinder. Berlin: Aufbau 2019.

\section{Sekundärliteratur:}

Frisé, Maria: Unterrichte meine Tochter, aber sieh sie nicht an! Aufwachsen in entsetzlichen Zeiten: Gusel Jachinas Roman "Wolgakinder". In: Frankfurter Allgemeine Zeitung vom 14.08.2019, unter: https://www.buecher.de/shop/ sowjetunion/wolgakinder/jachina-gusel/products_products/detail/prod_id/56247477/(abgerufen am 31.10.2020)

Funk, Victor/ Gusel Jachina: Autorin Gusel Jachina: „Das ist das Echo der Vergangenheit, das sich in uns zeigt". Interview in Frankfurter Rundschau, 6.05.2020, auch unter: https://www.fr.de/kultur/literatur/gusel-jachina-russlandstalin-echo-vergangenheit-zr-13750778.html (abgerufen am 1.11.2020)

Granzin, Katharina: Rezension in Büchermagazin, unter: https://www.buecher.de/shop/sowjetunion/wolgakinder/ jachina-gusel/products_products/detail/prod_ $\mathrm{id} / 56247477 /$ (abgerufen am 31.10.2020)

Großschupf, Johannes: Die Liebe in Sibirien. Der Tatarin Gusel Jachina ist mit „Suleika öffnet die Augen“ ein kraftvolles Debüt gelungen. In: Literaturkritik.de Rezensionsforum vom 4. April 2017, unter: https://iteraturkritik.de/ jachina-suleika-oeffnet-augen-liebe-sibirien-tatarin-guseljachina-ist-mit-suleika-oeffnet-augen-ein-kraftvolles-debuet-gelungen,23153.html (abgerufen am 1.11.2020)

Grützner, Annika: Ein Leben in der gewählten Einsamkeit. „Wolgakinder" von Gusel Jachina. In: Read Ost. Literatur Blog vom 23. Juni 2019, unter: https://read-ost.com/2020/06/ 23/ein-leben-in-der-gewahlten-einsamkeit-wolgakindervon-gusel-jachina/ (abgerufen am 1.11.2020) 
Hochweis, Olga: Das traurige Leben des Jakob Iwanowitsch Bach. Deutschlandfunk Kultur vom 1.10.2019, unter: https://www.deutschlandfunkkultur.de/gusel-jachina-wolgakinder-das-traurige-leben-des-jakob.950.de.html?dram: article_id=460032 (abgerufen am 31.10.2020)

Links, Christina (Moderation): Georg Dehio-Buchpreis 2020: Gusel Jachina spricht über ihren Roman ,Wolgakinder". YouTube-Sendung vom 16.11.2020, zu sehen unter: https://www.youtube.com/watch?v=T4IpPPTooHg (abgerufen am 1.11.2020)

o. A.: Keine Gnade in Gnadental: Gusel Jachinas «Wolgakinder». In: Westfälische Nachrichten vom 05.11.2019, unter: https://www.wn.de/Welt/Kultur/Buch/4020573Schicksalsschlaege-Keine-Gnade-in-Gnadental-GuselJachinas-Wolgakinder (abgerufen am 30.10.2020)

Zekri, Sonja: Im Kopf von Josef Stalin. Gusel Jachinas Roman „Wolgakinder“. In: Süddeutsche Zeitung vom 17.01.2020, unter: https://www.buecher.de/shop/sowjetunion/wolgakinder/jachina-gusel/products_products/detail/prod_ $\mathrm{id} / 56247477 / \#$ reviews (abgerufen am 1.11.2020) 